\title{
GUTER RAT
}

\section{Liebe Leserin, lieber Leser,}

wozu dient ein Beirat? Bei ihm holt man sich den sprichwörtlich guten Rat. Laut Wikipedia ist er ein Gremium mit beratender Funktion. Dieser Ausschuss soll eine Zielgruppe in ein Projekt einbinden, um auch den externen Sachverstand nutzen zu können. Die ATZ-Familie nutzt seit vielen Jahren die Vorteile eines aktiven Beirats, auch um den Berufenen ihre Wertschätzung zu zeigen.

In den letzten Wochen fanden die Sitzungen der technisch-wissenschaftlichen Beiräte von ATZ, ATZelektronik und MTZ statt. Einmal im Jahr treffen sich die Entwicklungsleiter von OEMs, Zulieferern und Entwicklungsdienstleistern sowie die Institutsleiter der Hochschulen, um aus Rückschau und Ausblick heraus die Leitplanken der redaktionellen Berichterstattung in unseren Fachzeitschriften $\mathrm{zu}$ justieren. Was war im vergangenen Jahr wichtig, was wird im nächsten wichtig werden? In Fulda, Sindelfingen und Stuttgart wurden diejenigen Themen diskutiert, die sich in unseren Zeitschriften publizistisch wiederfinden sollen. Dazu zählten die ATZ-Beiräte Dauerbrenner wie elektrische und Hybridantriebe, $\mathrm{CO}_{2}$-Minderung und Leichtbau sowie Jüngeres wie automatisiertes Fahren, bessere Aerodynamik bei Lkw durch Längenänderung und Connected Services (Inboard, Offboard), aber auch die Funktionalität von Bedienkonzepten. Wenn Sie wissen möchten, wer genau im Beirat der ATZ aktiv ist, informiert Sie das Impressum auf Seite 88.

Des Weiteren ging es dem Beirat um die Einheitlichkeit von Begriffen. Statt aktive und passive Sicherheit wird jetzt der Terminus integrale Sicherheit verwendet. Die beiden Abteilungen Berechnung/Simulation und Versuch (Messen, Testen, Prüfen) arbeiten schon lange nicht mehr unabhängig voneinander. Diese Einzelthemen gehören begrifflich zusammen; auf sie trifft besser der gemeinsame Begriff Testing zu. Die Redaktion ist dankbar für solche Anstöße und nimmt diese Impulse gern auf.

Zukünftig möchten wir den Beirat noch mehr einbinden. Die Teilnehmer werden vor dem nächsten Treffen gebeten, aus ihrer Sicht drei Topthemen zu nennen. Die anschließende Diskussion wird sicher spannende Ergebnisse zeigen, man denke nur an heute brisante Themen wie den NEFZ-Nachfolger, das Kältemittel R1234yf oder die anscheinend klare Begrifflichkeit, ob es nun autonomes oder automatisiertes Fahren heißt. Bei allen Meinungsverschiedenheiten auf der nächsten Beiratssitzung im Herbst 2014 sollte nicht nur der Disput, sondern auch der Ideenaustausch im Mittelpunkt stehen. Ein finnisches Sprichwort lautet: Guter Rat ist wie Schnee, je leiser er fällt, desto länger bleibt er liegen. Ich wünsche Ihnen allzeit guten Rat - und einen guten Start ins neue Jahr 2014.

Herzlichst, Ihr

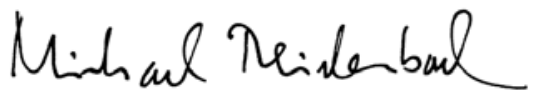

DIPL.-ING. MICHAEL REICHENBACH,

Stellvertretender Chefredakteur

Wiesbaden, 26. November 2013

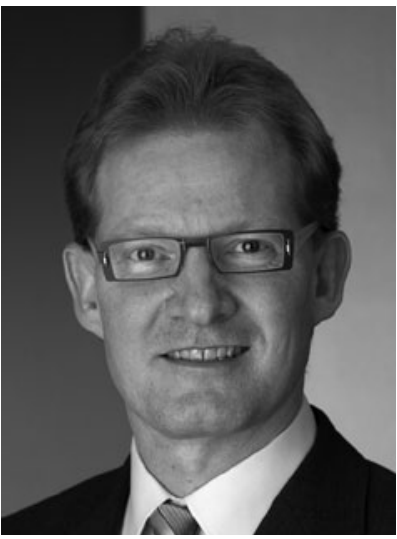

DYNA4 Advanced Powertrain

\section{Energiemanagement im Fahrzeug}

- Simulation von Einzelfahrzeugen bis hin zur Fahrzeugflotte

- Fahrleistungs- und Verbrauchsberechnungen

- Konventionelle Antriebe, Hybridund Elektrofahrzeuge

- Variantenhandling, Automation und Reporting

Mehr erfahren unter www.tesis-dynaware.com/flv 